\title{
Questes
}

\section{Publier la Bible durant les guerres de religion: Roville et les libraires lyonnais}

Ji Gao

\section{(2) OpenEdition}

\section{Édition électronique}

URL : http://journals.openedition.org/questes/4892

DOI : 10.4000/questes.4892

ISSN : 2109-9472

\section{Éditeur}

Les Amis de Questes

\section{Édition imprimée}

Date de publication : 1 juillet 2018

Pagination : 119-139

ISSN : 2102-7188

\section{Référence électronique}

Ji Gao, «Publier la Bible durant les guerres de religion : Roville et les libraires lyonnais », Questes [En ligne], 38 | 2018, mis en ligne le 18 juin 2018, consulté le 20 avril 2019. URL : http:// journals.openedition.org/questes/4892 ; DOI : 10.4000/questes.4892 


\title{
Publier la Bible durant les guerres de religion : Roville et les libraires lyonnais
}

\author{
Ji GAO \\ University of Chicago
}

Idéalement située à un carrefour commercial et culturel en Europe, Lyon s'est très vite dotée d'imprimeries au XVI ${ }^{e}$ siècle, devenant l'un des plus grands centres d'imprimerie de France ${ }^{1}$. Dans cette expansion du marché du livre, Lyon se distinguait à la fois par la quantité considérable de livres religieux publiés par les presses locales, et par sa position stratégique. Ville française sous le contrôle des autorités parisiennes, Lyon se situait à la périphérie du royaume et subissait par conséquent de fortes influences étrangères - notamment genevoises et italiennes - par le biais du commerce.

Lorsqu'il s'agissait de publier la Bible, les imprimeurs et libraires lyonnais étaient tiraillés entre la censure de Paris et la forte influence des presses protestantes de Genève, capitale du calvinisme depuis 1541. Tandis que dans les années 1550, le climat culturel était davantage tolérant et que les libraires lyonnais rééditaient des versions genevoises de la Bible française, les tensions s'accentuèrent considérablement dans les années 1560 , après la brève occupation de la ville par les réformés. Cette occupation, nommée aussi le Sac de Lyon, dura de la fin du mois

\footnotetext{
${ }^{1}$ Pour une présentation générale du milieu lyonnais des imprimeurs et des libraires au $\mathrm{XVI}^{\mathrm{e}}$ siècle, voir Natalie Zemon Davis, «Le monde de l'imprimerie humaniste: Lyon », dans Histoire de l'édition française, dir. Roger Chartier et Henri-Jean Martin, Paris, Promodis, 1983-1986, 4 vol., vol. 1, 1983, p. 255-277.
} 
d'avril 1562 jusqu'à mai $1563^{2}$. Ce fut une courte période durant laquelle il y eut beaucoup de destructions et de pillages.

La présente étude sur la publication de la Bible durant les guerres de religion est avant tout fondée sur des catalogues classiques: les bibliographies des livres imprimés à Lyon au $\mathrm{XVI}^{\mathrm{e}}$ siècle d'Henri et de Julien Baudrier ${ }^{3}$ ainsi que de Sybille von Gültlingen ${ }^{4}$, auxquelles il faut ajouter la bibliographie des Bibles françaises de Betty Chambers ${ }^{5}$. Plusieurs autres études nous fournissent des informations importantes sur ce sujet. Par exemple, l'article de Clive R. Sneddon ${ }^{6}$ présente le contexte général de publication de la Bible française au XVI ${ }^{\mathrm{e}}$ siècle. L'ouvrage de Victor Baroni ${ }^{7}$ contient des analyses concernant les rapports entre la Contre-Réforme et la Bible. L'étude de Robert Kingdon ${ }^{8}$ présente l'influence des presses genevoises sur la France, et la recherche de Francis Higman ${ }^{9}$ fournit une présentation détaillée sur la censure des

\footnotetext{
${ }^{2}$ Pour une présentation plus détaillée de la ville de Lyon durant cette période, voir Lyon 1562, capitale protestante. Une histoire religieuse de Lyon à la Renaissance, dir. Yves Krumenacker, Lyon, Olivétan, 2009; Aimé Vingtrinier, Histoire de l'imprimerie à Lyon de l'origine jusqu'à nos jours, Lyon, Storck, 1894.

${ }^{3}$ Henri et Julien Baudrier, Bibliographie lyonnaise. Recherches sur les imprimeurs, libraires, relieurs et fondeurs de lettres de Lyon au XVI siècle, Paris, F. de Noble, 1964, 12 vol. et 1 supplément.

${ }^{4}$ Sybille von Gültlingen, Bibliographie des livres imprimés à Lyon au XVI ${ }^{e}$ siècle, Baden-Baden/Bouxwiller, Valentin Koerner, 1992, 10 vol.

${ }^{5}$ Betty Thomas Chambers, Bibliography of French Bibles. Fifteenth and sixteenth century French language editions of the scriptures, Genève, Droz, 1983.

${ }^{6}$ Clive R. Sneddon, «The Bible in French », dans The New Cambridge History of the Bible, vol. 2, From 600 to 1450, dir. E. Ann Matter et Richard Marsden, New York, Cambridge University Press, 2012, p. 251-267.

${ }^{7}$ Victor Baroni, «Les versions pour le peuple», dans id., La Contre-Réforme devant la Bible. La question biblique, Lausanne, La Concorde, 1943, p. 301-354.

${ }^{8}$ Robert Kingdon, «The Flood Tide : books from Geneva », dans id., Geneva and the coming of the Wars of Religion in France (1555-1563), Genève, Droz, 1956, p. $93-$ 105.

${ }^{9}$ Francis Higman, Censorship and the Sorbonne. A bibliographical study of books in French censured by the Faculty of Theology of the University of Paris (1520-1551), Genève, Droz, 1979 ; id., Piety and the People. Religious printing in French (15111551), Aldershot, Scolar Press, coll. «St Andrews Studies in Reformation History », 1996.
} 
autorités parisiennes entre 1520 et 1551 . Quant aux activités des libraires lyonnais dans le contexte du XVI $\mathrm{XI}^{\mathrm{e}}$ siècle, des mémoires récents au sujet des institutions lyonnaises nous offrent des études de cas détaillées ${ }^{10}$. Plus largement, en ce qui concerne la religion et les libraires lyonnais en tant que catégorie sociale, la thèse de Natalie Zemon Davis ${ }^{11}$ constitue jusqu'à ce jour une référence incontournable.

Afin de mettre en évidence l'évolution de la politique éditoriale concernant la Bible durant les guerres de religion, cette étude se focalise sur la production d'un libraire emblématique: Guillaume Roville (vers 1518-1589) ${ }^{12}$. Originaire de Dolus en Touraine, Roville se forma chez Gabriele Giolito de' Ferrari à Venise ${ }^{13}$. Après s'être installé définitivement à Lyon en 1543, il fut engagé par le libraire italien Vincent Portonariis. Avec sa production considérable ${ }^{14}$ et en association avec de nombreux libraires, imprimeurs, illustrateurs, voire juristes, en France et

\footnotetext{
${ }^{10}$ Marion Chalvin, Jacques Sacon, imprimeur-libraire lyonnais du XVI siècle (14971529), mémoire de recherche, Université Lumière-Lyon 2/ENSSIB, 2011 ; MarieDominique Tamet, Les Senneton, marchands-libraires à Lyon au XVI siècle, mémoire de recherche, ENSSIB, 2011 ; Léa Constantin, Les Enjeux de la controverse religieuse dans l'imprimerie lyonnaise (1560-1565), mémoire de recherche, Université Lumière Lyon 2/ENSSIB, 2011 ; Émeline Huguet, Macé Bonhomme, un imprimeur lyonnais du XVI $I^{e}$ siècle, mémoire de master 1, Université Lumière-Lyon 2/ENSSIB, 2013 ; Léa Constantin, Enjeux et bouleversements de l'imprimerie lyonnaise à la fin $d u$ XVI ${ }^{e}$ siècle. Le cas du libraire Jean Pillehotte, mémoire de recherche, ENSSIB, 2013 ; Bastien Rissoan, Jean Temporal, libraire de la Renaissance lyonnaise (1549-1571), mémoire de master 1, Université Lumière-Lyon 2/ENSSIB, 2013 ; Morgane Perrier, Thibaud Payen, imprimeur lyonnais du XVI siècle. Les premières années (15301545), Université Lumière-Lyon 2/ENSSIB, mémoire de master 2 professionnel, 2015 ; Louise Amazon, Remettre en lumière le catalogue d'un libraire à ses débuts. Benoît Rigaud (1555-1570), de l'étal au virtuel, mémoire de recherche, ENSSIB, 2017.

${ }^{11}$ Natalie Zemon Davis, Protestantism and the printing workers of Lyon. A study in religion and social class, University of Michigan, 1959.

${ }^{12}$ Ce libraire est aussi connu sous le nom de Guillaume Rouillé.

${ }^{13}$ Henri et Julien Baudrier, Bibliographie lyonnaise, op. cit., vol. 9, p. 13-124.

${ }^{14}$ Sybille von Gültlingen, Bibliographie des livres imprimés à Lyon au XVI siècle, op. cit. Gültlingen donne dans son catalogue un total de 1022 titres publiés par Roville, et de 36 par ses héritiers jusqu'en 1600.
} 
ailleurs en Europe, Roville parvint à se créer une grande clientèle ${ }^{15}$. Son érudition, ses goûts littéraires et sa politique éditoriale, son sens avisé du marché, ainsi que son souci d'une production de qualité firent de Roville l'un des libraires lyonnais les plus importants, au même titre que Jean I de Tournes ${ }^{16}$. Ses très nombreuses publications et sa très longue carrière professionnelle, qui s'étendit des années 1540 jusqu'aux années 1580, en font une figure particulièrement intéressante. L'examen de ce cas spécifique permettrait une mise en perspective de la façon dont les guerres de religion exercèrent une influence sur sa politique éditoriale.

Les premières présentations générales à propos de Roville, outre la notice figurant dans la bibliographie d'Henri et de Julien Baudrier, furent l'article de Natalie Zemon Davis ${ }^{17}$ puis, beaucoup plus tard, le mémoire d'Ilaria Andreoli ${ }^{18}$. Baudrier, en dépouillant les documents d'archives, rassemble les faits de sa biographie à travers une présentation chronologique. Zemon Davis s'intéresse davantage à la situation de Roville en tant que marchand-libraire et souligne les soucis commerciaux et personnels que reflète sa politique éditoriale. En adoptant une approche thématique, Andreoli s'appuie sur les documents d'archives et les pièces liminaires pour approfondir l'analyse des marchés et du public visés par l'immense production éditoriale de Roville.

\footnotetext{
${ }^{15}$ Henri et Julien Baudrier, Bibliographie lyonnaise..., op. cit., vol. 9.

${ }^{16}$ Imprimeur-libraire éminent qui a travaillé avec les plus grands poètes lyonnais de son temps, dont Maurice Scève, Louise Labé, Olivier de Magny, et qui a fait paraître un grand nombre d'ouvrages littéraires et religieux. Fondateur d'une dynastie d'imprimeurs-libraires à Lyon puis à Genève, ses héritiers comptent son fils Jean II de Tournes et son petit-fils Jean III de Tournes. Voir la notice biographique d'Eugène Vial, «Les de Tournes à Lyon », dans Alfred Cartier, Bibliographie des éditions des de Tournes, imprimeurs lyonnais, Paris, Éditions des bibliothèques nationales de France, 1937, p. 113-157.

${ }^{17}$ Natalie Zemon Davis, « Publisher Guillaume Rouillé, businessman and humanist », dans Editing Sixteenth-Century Texts, dir. Richard Schoeck, Toronto, University of Toronto Press, 1966.

${ }^{18}$ Ilaria Andreoli, Guillaume Rouillé (1518-1589). Libraire lyonnais, marchand et homme de lettres. Un esprit de la Renaissance ouvert à l'Europe, Lyon, Université Lumière-Lyon 2, 2000.
} 
En ce qui concerne plus spécifiquement les livres illustrés, Ilaria Andreoli a montré que beaucoup de gravures utilisées par Roville sont inspirées de modèles italiens et des pays du Nord, notamment du vénitien Gabriele Giolito de' Ferrari. Par ailleurs, son $\operatorname{article~}^{19}$ et celui de Jean $\mathrm{Dubu}^{20}$, paru deux décennies auparavant, ont présenté en détail les divers aspects historiques et artistiques du Promptuaire des medailles.

Quant à ses publications en langues vernaculaires étrangères, Émile Picot avait très tôt dédié une section à Roville dans son étude sur les Français italianisants ${ }^{21}$. Ces dernières années, Jean Balsamo a montré que les lettrés lyonnais, notamment Roville, avaient été très influencés par les auteurs italiens ${ }^{22}$. Dans son récent ouvrage sur le milieu éditorial lyonnais entre 1536 et $1551^{23}$, Élise Rajchenbach-Teller a dédié un chapitre, intitulé « Guillaume Rouillé sous l'écu de Venise », à un Roville en début de carrière et profondément marqué par l'influence italienne. Cette influence fut exercée tant par ses collaborateurs que par les autres publications dont il avait connaissance. Par ailleurs, l'étude de Noël

${ }^{19} I d$., «La storia in soldoni : il Promptuaire des medailles di Guillaume Rouillé », dans Storia per parole e per immagini. Actes du colloque de Cividale del Friuli (2003), Udine, Mino Gabriele, coll. « Libri e biblioteche », 2006, p. 235-266.

${ }^{20}$ Jean Dubu, «Le Promptuaire des medalles de Guillaume Rouillé », dans Il Rinascimento a Lione. Atti del congresso internazionale (Macerata, 6-11 maggio 1985), Roma, G. M. Antoni Possenti, 1988, p. 185-219.

${ }^{21}$ Émile Picot, Les Français italianisants au XVI e siècle, Paris, Champion, 1906. Pour ses études sur les rapports culturels entre la France et l'Italie au XVI siècle, voir encore id., Pour et contre l'influence italienne en France au XVI siècle, Paris, Ernest Leroux, 1920; id., Les Italiens en France au XVI siècle [1901], Roma, Vecchiarelli, 1995.

22 Jean Balsamo, «L'italianisme lyonnais et l'illustration de la langue française », dans Lyon et l'illustration de la langue française à la Renaissance, dir. Gérard Defaux, Lyon, ENS Éditions, 2003, p. 211-229. Pour l'influence italienne sur la littérature française, voir encore Jean Balsamo, Les Rencontres des muses. Italianisme et anti-italianisme dans les lettres françaises de la fin du XVI siècle, Genève, Slatkine, 1992.

${ }^{23}$ Élise Rajchenbach-Teller, « Mais devant tous est le Lyon marchant ». Construction littéraire d'un milieu éditorial et livres de poésie française à Lyon (1536-1551), Genève, Droz, 2016. 
Salomon a offert un aperçu des publications de Roville en espagnol et de ses rapports avec l'outre-Pyrénées ${ }^{24}$.

Plusieurs chercheurs se sont intéressés à la politique éditoriale de Roville. Citons notamment l'étude récente d'Élise Rajchenbach-Teller, selon laquelle Roville travailla activement à son image de passeur de textes $^{25}$. Dans la même lignée, l'article d'Elsa Kammerer, Aude Plagnard et Élise Rajchenbach-Teller examine les activités de Roville dans une perspective européenne et souligne que ce dernier, grâce à ses publications plurilingues, était soucieux de promouvoir les langues romanes comme langues littéraires ${ }^{26}$.

Il est remarquable que la Bible et le Nouveau Testament représentent une part considérable des publications de Roville. Si des travaux précédents avaient fait mention des publications religieuses du libraire et évoqué les rapports de ces dernières avec la Réforme ${ }^{27}$, il n'existait pas jusqu'ici d'étude prenant en compte la totalité de la carrière de Roville. Un examen spécifique de cet aspect important de sa publication pourrait éclaircir quelques points sur sa politique éditoriale tout au long des décennies tumultueuses du XVI $\mathrm{XI}^{\mathrm{e}}$ siècle et, plus largement,

\footnotetext{
${ }^{24}$ Noël Salomon, «Les éditions en langue espagnole d'un libraire lyonnais du $\mathrm{XVI}^{\mathrm{e}}$ siècle : Guillaume Rouillé », dans Imprimerie, commerce et littérature. Actes $d u$ $5^{e}$ Congrès national de littérature comparée (Lyon, 1962), éd. Société française de Littérature générale et comparée, Lyon, Audin, 1965, p. 61-73.

${ }^{25}$ Élise Rajchenbach-Teller, «De "ceux qui de leur pouvoir aydent et favorisent au publiq" : Guillaume Rouillé, libraire à Lyon », dans Passeurs de textes. Imprimeurs et libraires à l'âge de l'humanisme, dir. Christine Bénévent, Annie Charon, Isabelle Diu et Magali Vène, Paris, École nationale des chartes, coll. «Études et rencontres de l'École des chartes », 2012, p. 99-114.

${ }^{26}$ Elsa Kammerer, Aude Plagnard, Élise Rajchenbach-Teller, «Entre stratégies commerciales et "illustration" des vulgaires romans : la boutique de Guillaume Roville à Lyon (1548-1556)», dans Imprimeurs et libraires de la Renaissance. Le travail de la langue, éd. Elsa Kammerer et Jan-Dirk Müller, Genève, Droz, 2015, p. 443-455.

27 Voir en particulier Natalie Zemon Davis, «Publisher Guillaume Rouillé, businessman and humanist », art. cit. ; Ilaria Andreoli, Guillaume Rouillé (15181589)..., op. cit.
} 
sur les interactions entre l'activité éditoriale et les transformations de la pensée religieuse à la Renaissance.

À travers l'industrie de l'édition lyonnaise et l'exemple de Roville, le présent article se propose d'étudier la manière dont la recherche de la suprématie politico-religieuse et les fortunes changeantes des catholiques et des réformés ont contribué au renforcement progressif du sentiment d'appartenance confessionnelle. Après une présentation des enjeux de la publication de la Bible, nous nous pencherons sur les facteurs externes qui ont influencé la religiosité lyonnaise, de manière à pouvoir ensuite procéder à l'examen de la politique éditoriale de Roville dans une perspective évolutive.

\section{Les traductions de la Bible et les choix des libraires lyonnais}

La traduction de la Bible était un travail philologique au cœur des enjeux politico-religieux à une époque de remise en question de l'autorité de l'Église catholique. La Vulgate connut plusieurs révisions et quelques nouvelles traductions latines furent réalisées à partir de l'hébreu et du $\operatorname{grec}^{28}$. On observe que ces traductions avaient «tendance à s'écarter de plus en plus de la Vulgate ${ }^{29} »$. L'édition la plus célèbre fut celle du Nouveau Testament par Érasme, parue à Bâle en 1516, qui offre en parallèle au texte grec une nouvelle traduction latine. Cette traduction fut plus tard un texte de référence inestimable pour les réformateurs.

La Bible historiale ${ }^{30}$ et la Bible abrégée $e^{31}$ étaient les éditions françaises les plus répandues avant les années 1520. Jacques Lefèvre

\footnotetext{
${ }^{28}$ Jean-Pierre Delville, «L'évolution des vulgates et la composition de nouvelles versions latines de la Bible au XVI siècle », dans Biblia. Les Bibles en latin au temps des Réformes, dir. Marie-Christine Gomez-Géraud, Paris, Presses de l'Université Paris-Sorbonne, 2008, p. 71-106.

${ }^{29}$ Ibid., p. 99.

${ }^{30}$ Clive R. Sneddon, « The Bible in French », art. cit., p. 257.

${ }^{31}$ Betty Thomas Chambers, Bibliography of French Bibles..., op. cit., p. 1.
} 
d'Étaples ${ }^{32}$ contribua à la diffusion de l'Écriture avec sa nouvelle traduction française du Nouveau Testament et de la Bible intégrale ${ }^{33}$ qui, à partir des années 1520, connut de nombreuses rééditions. En 1535, la traduction de Pierre Robert Olivétan ${ }^{34}$ fut imprimée par Pierre de Vingle ${ }^{35}$ à Serrières près de Neuchâtel, dans une ville gagnée par la Réforme en 1530 sous l'impulsion de Guillaume Farel $^{36}$. Cette première Bible réformée ${ }^{37}$ en langue française fut la source de toutes les versions dites « de Genève », en particulier de celle publiée par Jean Girard en $1540^{38}$. Comme nous le verrons à travers l'étude de la production de Roville et des autres libraires lyonnais, la publication de la Bible genevoise fut particulièrement répandue au milieu du siècle. Cette situation dura jusqu'au milieu des années 1560 , lorsque les guerres de religion mirent un frein à la diffusion des versions genevoises à Lyon.

\section{Le contexte politico-religieux : le Concile de Trente et les influences de Paris et de Genève}

Afin de comprendre la situation des libraires lyonnais durant les guerres de religion, plusieurs facteurs doivent être examinés de plus près :

\footnotetext{
${ }^{32}$ Guy Bedouelle, Lefèvre d'Étaples et l'intelligence des Écritures, Genève, Droz, 1976 ; Philip E. Hughes, Lefèvre, pioneer of ecclesial renewal in France, Grand Rapids, Eerdmans, 1984.

${ }^{33}$ Traduction française faite à partir de la Vulgate, mais avec des corrections d'après les originaux grecs.

${ }^{34}$ La Bible Qui est toute la Saincte escripture... translatez en Francoys..., Neuchâtel, Pierre de Vingle, 1535. Selon Chambers, la traduction a été faite à partir de l'hébreu et du grec, mais Olivétan a également fait référence aux autres traductions, latines ou françaises, de son temps. Au sujet d'Olivétan, voir Olivétan, traducteur de la Bible, dir. Georges Casalis et Bernard Roussel, Paris, Éditions du Cerf, 1987.

${ }^{35}$ Eugénie Droz, «Pierre de Vingle, l'imprimeur de Farel», dans Aspects de la propagande religieuse, Genève, Droz, coll. "Travaux d'humanisme et Renaissance », 1957, p. 38-78.

${ }^{36}$ Voir Histoire du pays de Neuchâtel, éd. Société d'histoire et d'archéologie du canton de Neuchâtel, Hauterive, Attinger, 1989-1993, 3 vol.

${ }^{37}$ Selon Chambers, Jean Calvin a contribué à trois préfaces de cette édition.

${ }^{38}$ La Bible en laquelle sont contenus tous les livres canoniques... translaté en langue françoise..., Genève, Jean Girard, 1540.
} 
les réformes adoptées lors du Concile de Trente, la censure des autorités basées à Paris, et l'influence des livres réformés en provenance de Genève, foyer calviniste situé à proximité.

L'événement le plus emblématique de la mouvance de la ContreRéforme fut le Concile de Trente, dont les trois sessions se sont déroulées de 1545 à $1563^{39}$. Au cours de ce concile, de nombreuses réformes furent adoptées en matière de discipline et de dogme ${ }^{40}$. Face à la sola scriptura ${ }^{41}$ de Calvin, pour qui l'Écriture était «le fondement exclusif de la vérité et de la vie chrétienne ${ }^{42} »$, le Concile de Trente réaffirma l'autorité de la Vulgate $^{43}$ et déclara que l'Écriture devait également être complétée par «les traditions non-écrites » dites « apostoliques $^{44}$ ». Quant à la traduction de la Bible en langue vernaculaire, le concile demanda que toute édition de la Bible, quelle que fût la langue choisie, soit examinée par l'autorité ecclésiastique avant d'être mise en circulation ${ }^{45}$. La censure fut aussi renforcée à la suite de la parution en 1558 du premier Index librorum prohibitorum $^{46}$. À Lyon, les Jésuites, promoteurs de la Contre-Réforme, se virent confier dès 1565 le collège de la Trinité ${ }^{47}$.

39 Robert Bireley, «Redefining Catholicism: Trent and beyond», dans The Cambridge history of Christianity, vol. 6, Reform and expansion (1500-1660), Cambridge, Cambridge University Press, 2008, p. 143-161.

${ }^{40}$ Ibid.

${ }^{41}$ Pour la théologie protestante, voir Mark Greengrass, «The theology and liturgy of Reformed Christianity », dans The Cambridge history of Christianity, vol. 6, op. cit., p. 104-124.

${ }^{42}$ Olivier Millet, «Les églises réformées », dans Histoire du christianisme, vol. 8, Le temps des confessions (1530-1620/30), dir. Marc Vénard, Paris, Desclée, 1992, première partie, p. 55-117, cit. p. 72.

${ }_{43}$ Marc Vénard, «L'Église catholique », dans Histoire du christianisme, vol. 8, op. cit., p. 223-280.

${ }^{44}$ Ibid., p. 242.

${ }^{45}$ Ibid.

${ }^{46}$ Index librorum prohibitorum..., Roma, Paulo Manuzio, 1564.

${ }^{47}$ Pour connaître l'histoire de cette institution, voir Georgette de Groër, Réforme et Contre-Réforme en France. Le collège de la Trinité au XVI siècle à Lyon, Paris, Publisud, 1995. 
Grâce à la présence de l'Université et à son statut de capitale du royaume de France, Paris était au XVI siècle la ville la plus peuplée à l'échelle européenne ${ }^{48}$. La prééminence de Paris étant politique et religieuse, les libraires lyonnais devaient faire face à la censure exercée par les autorités parisiennes ${ }^{49}$.

Le 28 août 1525, le Parlement de Paris ${ }^{50}$ et la Faculté de Théologie ${ }^{51}$ interdirent la traduction vernaculaire du Nouveau Testament de Jacques Lefèvre d'Étaples, parue la même année. Dès lors, aucune traduction du Nouveau Testament ne fut plus imprimée à Paris jusqu'en $1565^{52}$. En 1551 fut promulgué l'Édit de Châteaubriant qui, face à la prolifération des écrits calvinistes en provenance de Genève, interdit expressément l'impression et la vente de nouvelles traductions de la Bible ou des écrits des Pères de l'Église sans l'approbation préalable de la Faculté de Théologie. La ville de Lyon, particulièrement susceptible de

\footnotetext{
${ }^{48}$ Pour la présentation de Paris au XVI ${ }^{\mathrm{e}}$ siècle, voir Jean Jacquart, « Paris : first metropolis of the early modern period », dans Capital cities and their hinterlands in early modern Europe, dir. Peter Clark et Bernard Lepetit, Aldershot, Scolar Press, coll. «Historical urban studies », 1996, p. 104-118; Jean-Pierre Babelon, Paris au XVI $I^{e}$ siècle, Paris, Hachette, 1986.

${ }^{49}$ Par ailleurs, ces deux institutions devaient également respecter la volonté du roi qui, à partir du concordat de Bologne en décembre 1516, était pourvu du pouvoir de nommer les évêques.

${ }^{50}$ Pour le rôle du Parlement dans la politique française, voir Roger Doucet, Étude sur le gouvernement de François $I^{e r}$ dans ses rapports avec le Parlement de Paris, Paris, Champion, 1921.

${ }^{51}$ Pour une présentation du rôle de la Faculté de Théologie de Paris dans la première moitié du XVI ${ }^{\mathrm{e}}$ siècle, voir James Farge, Orthodoxy and Reform in early reformation France. The Faculty of Theology of Paris (1500-1543), Leiden/Boston, Brill, coll. «Studies in medieval and reformation thought », 1985.

52 À l'exception du Nouveau Testament imprimé par Simon Du Bois en 1525 après le décret du Parlement condamnant la traduction de Lefèvre. Il est à noter que ce Nouveau Testament n'a pas obtenu de privilège, ce qui témoigne de l'impact du décret. $C f$. Higman, Censorship and the Sorbonne..., op. cit., p. 78.
} 
subir l'influence réformée, fut dès lors soumise à un contrôle très strict : il était prévu que les librairies soient inspectées trois fois par $\mathrm{an}^{53}$.

Ces soupçons n'étaient pas sans fondement. Principal foyer calviniste situé juste en-dehors du royaume français, Genève formait de nombreux pasteurs avant de les envoyer auprès des nouvelles communautés en France. Les livres sortis des presses genevoises exerçaient par ailleurs une influence considérable en France ${ }^{54}$. Cette influence se propageait habituellement le long du Rhône, jusqu'à Lyon, ville à laquelle Genève était étroitement liée par le commerce ${ }^{55}$. Les nombreuses presses de Genève ${ }^{56}$ étaient sous le patronage et le contrôle des autorités municipales qui exerçaient une censure stricte sur tous les aspects de la publication de livres imprimés ${ }^{57}$.

Ces observations préliminaires, concernant la spécificité de la Bible imprimée et l'influence des foyers catholiques et protestants, sont essentielles pour expliquer la politique éditoriale des libraires lyonnais au cours de cette période. Afin de mieux comprendre l'influence des troubles religieux sur la production des libraires lyonnais, nous procédons à l'étude du cas de l'un des principaux éditeurs actifs à Lyon au XVI ${ }^{e}$ siècle : Guillaume Roville. On peut distinguer deux phases majeures dans la publication de la Bible chez Roville : avant et après 1562. En effet, il commença par publier des Nouveaux Testaments vernaculaires, avant de se tourner cette année-là vers l'édition de Bibles latines et de

\footnotetext{
${ }^{53}$ Les Ordonnances et edicz faictz par le Roy treschretien Henri deuxiesme..., Paris, Sertenas, 1557, fol. $\mathrm{I}^{\circ} \mathrm{v}^{\circ}$ sq., section XVII. Cité par Francis Higman, Lire et découvrir. La circulation des idées au temps de la Réforme, Genève, Droz, 1998, p. 121-122.

${ }^{54}$ Robert Kingdon, «The Flood Tide: books from Geneva», art. cit. ; Andrew Pettegree, The French book and the European book world, Leiden/Boston, Brill, coll. «Library of the written word - The handpress world », 2007, p. 89-106.

${ }^{55}$ Paul-Frédéric Geisendorf, «Lyon et Genève du XVI ${ }^{\mathrm{e}}$ au XVIII" siècle : les foires et l'imprimerie », Cahiers d'histoire, vol. 5, n¹, 1960, p. 65-67.

${ }^{56}$ Robert Kingdon, «The Flood Tide : books from Geneva », art. cit.

${ }^{57}$ Ibid.
} 
Bibles illustrées. Nous étudierons successivement ces deux productions dans cette partie et la suivante, avant de nous pencher sur le cas des Bibles illustrées.

\section{Le Nouveau Testament vernaculaire et la politique éditoriale de Roville avant 1562}

Depuis les années 1540 jusqu'en 1562, la politique éditoriale de Roville, qui fut de confession catholique durant toute sa vie, se distinguait, concernant la Bible, par le nombre d'éditions du Nouveau Testament en français comme l'indique le graphique ci-dessous :

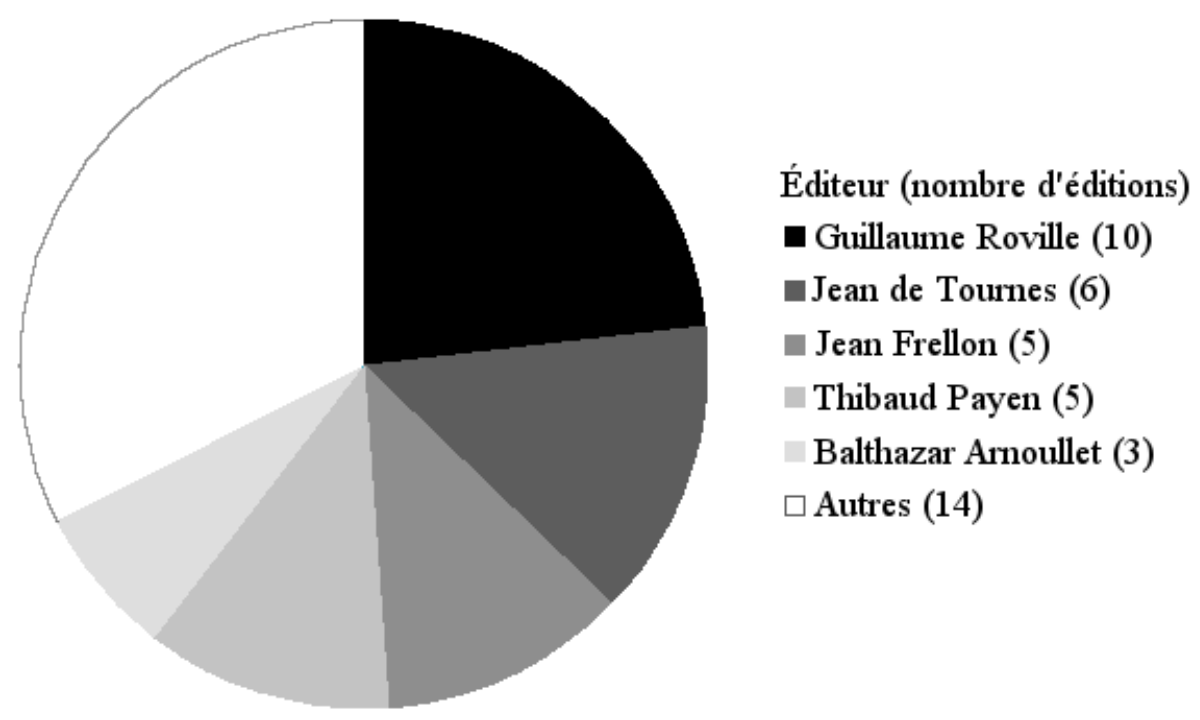

Nouveaux Testaments en français publiés à Lyon entre 1540 et 1562.

Ce graphique est fondé sur la recension de Betty Thomas Chambers.

On peut observer que le catholique Roville était à cette époque le principal producteur d'éditions du Nouveau Testament en français ${ }^{58}$. Si le lectorat catholique restait très important, il faut prendre en considération que c'étaient les réformés qui encourageaient la circulation des textes bibliques en langue vernaculaire. En effet, les autres libraires ayant

\footnotetext{
${ }^{58}$ Et cela sans tenir compte des Nouveaux Testaments en italien publiés par Roville avant 1562 : les éditions de Il Nuovo Testamento di Giesu Christo salvatore nostro parues en 1547, 1549, 1550, 1552, 1553, 1558, et celle de L'Epistole di San Paulo Apostolo parue en 1558.
} 
imprimé ce texte (en particulier Jean I de Tournes ${ }^{59}$, Jean II Frellon ${ }^{60}$, Thibaud Payen ${ }^{61}$ et Balthazar Arnoullet ${ }^{62}$ ) étaient explicitement réformés ou avaient au moins des affinités avec les nouvelles croyances.

Roville avait même contribué à la première impression à Lyon d'une Bible genevoise ${ }^{63}$ en 1544. Il se chargea par ailleurs de la publication d'une Bible intégrale en français, publiée en $1547^{64}$ et $1548^{65}$, qui s'appuyait sur la Bible genevoise de 1544. Cette version de la Bible, publiée la même année par Jean Pidier et Nicolas Bacquenois ${ }^{66}$, condamnait et recommandait en même temps les livres non-canoniques de la Bible, afin de concilier la censure catholique et les sympathies réformées. Cette attitude ambiguë révèle les hésitations des libraires lyonnais de l'époque, lorsque les nouvelles croyances étaient en voie de s'établir. La politique éditoriale de Roville témoigne donc d'une grande flexibilité et pourrait résulter des calculs économiques de ce marchandlibraire.

Les Nouveaux Testaments publiés par Roville étaient essentiellement fondés sur l'édition genevoise de Jean Girard, parue en

\footnotetext{
${ }^{59}$ Pour la conversion de Jean I de Tournes à la Réforme, voir la notice biographique d'Eugène Vial, «Les de Tournes à Lyon », art. cit.

${ }^{60}$ Pour la contribution active de Jean II Frellon à la diffusion des idées calvinistes et son lien avec Calvin, voir Yves Krumenacker, «Le livre religieux à Lyon au $\mathrm{XVI}^{\mathrm{e}}$ siècle (1517-1561)», Bulletin des bibliothèques de France, 2007, p. 2031.

${ }^{61}$ Henri et Julien Baudrier, Bibliographie lyonnaise..., op. cit., vol. 4, p. 207. Selon Baudrier, le nom de Thibaud Payen figure dans une liste de «Huguenotz reduictz » datant de 1569, preuve que Payen se serait déjà converti au calvinisme auparavant.

${ }^{62}$ Ibid., vol. 10, p. 91-148. Â la suite de son impression à Vienne (Dauphiné) en 1552-1553 de la Christianismi restitutio d'un Michel Servet condamné par l'Inquisition, Arnoullet fut poursuivi par la justice et emprisonné.

${ }^{63}$ Gérard Defaux, «À l'Enseigne du Rochier : Étienne Dolet, Guillaume Rouillé et la Bible "Sabon/Constantin" de 1544 », French Forum, n' 22 (1), 1997, p. 540.

${ }^{64}$ La Bible en Francoys... Recentement reveuz et fidelement corrigez selon l'Ebrieu, Grec et Latin, Lyon, Guillaume Rouillé et Thibaud Payen, 1547.

${ }^{65}$ La Bible en Francoys... Recentement reveuz et fidelement corrigez selon l'Ebrieu, Grec et Latin, Lyon, Guillaume Rouillé et Thibaud Payen, 1548. Il s'agit d'une réimpression de l'édition publiée l'année précédente.

${ }^{66}$ La Bible en Francoys..., Lyon, Jean Pidier et Nicolas Bacquenois, 1547.
} 
1543 et revue par Calvin lui-même ${ }^{67}$. Plusieurs remarques générales peuvent être faites. D'abord, toutes étaient de petit format (in-16), tout comme celles de Jean I de Tournes, ce qui suppose un usage personnel, par des religieux ou des laïcs, plutôt que liturgique. Ensuite, les résumés des chapitres dans les éditions lyonnaises de 1545 d'Arnoullet, de Roville et de Jean de Tournes sont calqués sur les «Indice des chapitres » de la version de Girard. Enfin, l'absence du nom de Calvin dans une version séparée de la même édition de Girard signifie qu'elle était destinée à être diffusée en France.

La publication des Nouveaux Testaments français était donc bien l'indice d'une sympathie ou, tout du moins, d'une ouverture d'esprit à l'égard des idées nouvelles, d'autant plus que les éditions lyonnaises de ce texte étaient d'inspiration genevoise. Comme Jean I de Tournes, Roville fut l'un des libraires les plus actifs dans la diffusion de l'Écriture en vernaculaire. Durant la seule année 1554, Roville fit paraître trois éditions du Nouveau Testament français. Selon Francis Higman, les Bibles genevoises étaient principalement de petit format et contenaient le texte biblique traduit, souvent associé aux Psaumes ou au catéchisme de Calvin $^{68}$. Bien qu'ils ne mentionnent pas le nom de Calvin, les Nouveaux Testaments de Roville, dont le contenu était repris de l'édition genevoise de Girard, présentaient par conséquent une politique éditoriale similaire à celle des calvinistes.

\footnotetext{
${ }^{67}$ Selon Chambers, Roville publia en 1545 son tout premier Nouveau Testament qui était une autre version de celui publié la même année par Balthazar Arnoullet. Cette édition d'Arnoullet se fondait elle-même sur celle publiée par Jean Girard à Genève en 1543, qui fut le premier Nouveau Testament révisé par Calvin. Voir la notice du catalogue de Betty Thomas Chambers, Bibliography of French Bibles..., op. cit., $\mathrm{n}^{\circ} 126$ : Le Nouveau Testament... Translaté de Grec en François, Reveu par M. Jehan Calvin, Genève, Jean Girard, 1543.

${ }^{68}$ Francis Higman, "Without great effort, and with pleasure", Sixteenth-century Genevan Bibles and Reading Practices », dans The Bible as book. The Reformation, dir. Orlaith O'Sullivan, London, The British Library/Oak Knoll Press, 2000, p. 115122, voir en particulier p. 116-117.
} 


\section{La Bible latine et la politique éditoriale de Roville après 1562}

En revanche, à partir de 1562 et jusqu'à la fin de sa carrière, Roville ne publia plus de Nouveau Testament en langue vernaculaire. Sa politique éditoriale contraste avec celle de Jean I de Tournes qui continua à publier des Nouveaux Testaments en 1562 et en 1563, durant la courte période de l'occupation de Lyon par les réformés. Le contexte favorable aux croyances nouvelles fit que de Tournes, ne craignant plus la censure, osa mettre sur la page de titre de ces éditions «Reveu de nouveau et corrigé sur le Grec par l'advis des Ministres de Geneve. Avec annotations reveuës et de nouveau augmentees ${ }^{69}{ }$, tout comme les éditions publiées à Genève même. L'année 1562 marqua donc le point de rupture entre deux types de politiques éditoriales à Lyon, représentées par Guillaume Roville et par Jean I de Tournes.

Ainsi les tensions entre catholiques et protestants entravèrent en partie la production de Bibles vernaculaires. La responsabilité politique de Roville, capitaine pennon ${ }^{70}$ de son quartier ${ }^{71}$, le rendait particulièrement sensible au changement de climat politico-religieux. Le Nouveau Testament latin de petit format fut lui aussi remplacé par la Bible intégrale latine de grand format à partir de 1563. La période qui suit correspond à la seconde phase de la publication de la Bible par Roville. Il fit paraître neuf

${ }^{69}$ Le Nouveau Testament... Reveu de nouveau et corrigé sur le Grec par l'advis des Ministres de Geneve..., Lyon, Jean de Tournes, 1562 ; Le Nouveau Testament... Reveu de nouveau et corrigé sur le Grec par l'advis des Ministres de Genéve..., Lyon, Jean de Tournes et Guillaume Gazeau, 1563 ; Le Nouveau Testament... Reveu de nouveau et corrigé sur le Grec, par l'advis des Ministres de Genéve..., Lyon, Jean de Tournes et Guillaume Gazeau, 1563. Cette copie contient des «arguments » pour tous les livres.

${ }^{70}$ Le pennonage est un système de définition de quartier à Lyon au XVI ${ }^{\mathrm{e}}$ siècle. Le capitaine pennon était l'un des commandants d'un pennonage. Voir Olivier Zeller, «Les quartiers d'affaires à Lyon au XVI ${ }^{\mathrm{e}}$ siècle », dans Or, monnaie, échange dans la culture de la Renaissance. Actes du $9^{e}$ Colloque international de l'Association Renaissance, Humanisme, Réforme, dir. André Tournon et Gabriel-André Pérouse, Saint-Étienne, Publications de l'Université de Saint-Étienne, 1994, p. 31-42.

${ }^{71}$ Henri et Julien Baudrier, Bibliographie lyonnaise..., op. cit., vol. 7, p. 74. 
éditions au total de la Biblia sacra entre 1562 et 1588, c'est-à-dire qu'il publia ce texte jusqu'à sa mort en 1589. L'impression de la Bible intégrale latine correspond à l'affirmation de l'authenticité de la Vulgate prônée par le Concile de Trente.

De ces neuf éditions, quatre sont in-octavo (in-8), un format facile à porter pour le lecteur. Les cinq autres sont en grand format in-folio (in2), et donc très probablement destinées à être posées sur des lutrins pour la liturgie ou dans les bibliothèques des établissements religieux. Par exemple, l'édition de 1566, qui compte plus de mille pages, est un épais in-folio annoté et illustré. Sa page de titre est également ornée d'une belle gravure. Un exemplaire de cette édition, actuellement conservé à la Bibliothèque municipale de Lyon, a appartenu au Collège de la Sainte Trinité de la Compagnie de Jésus de Lyon à partir de 1612, ce qui signifie qu'il fut très probablement utilisé par les Jésuites ${ }^{72}$.

Il faut souligner que la publication des Bibles vernaculaires continua à fleurir à Lyon jusqu'en $1567^{73}$. Selon Chambers, on observe qu'après 1567, certains libraires choisirent de continuer à publier le texte de la Bible tout en supprimant les éléments d'origine genevoise, afin de donner une apparence plus « orthodoxe» à leur production. L'autre solution était de publier des éditions bilingues latin-français, une combinaison de l'édition genevoise et de la Vulgate, pour échapper à la censure.

Cependant, sentant le vif danger de faire de la Bible vernaculaire l'apanage des réformés, les catholiques décidèrent de promouvoir une version catholique de ce texte. En 1550 fut publiée une Bible approuvée

\footnotetext{
${ }^{72}$ D'après le catalogue en ligne de la Bibliothèque municipale de Lyon (site de la Part-Dieu, Silo ancien, cote 20047).

${ }^{73}$ Betty Thomas Chambers, Bibliography of French Bibles..., op. cit., p. 350-420.
} 
par les docteurs en théologie de l'Université de Louvain ${ }^{74}$. Le cotraducteur

Nicolas de Leuze attaque la conviction réformée de la sola scriptura dans sa préface au lecteur ${ }^{75}$. Citons aussi la traduction de la Bible par le docteur en Sorbonne René Benoist ${ }^{76}$, soutenue par les docteurs de Louvain, mais attaquée par ses collègues parisiens. Benoist fut exclu de la Sorbonne en 1572 et sa Bible officiellement interdite par le pape en $1575^{77}$. En 1578, son texte est pourtant imprimé par Christophe Plantin à Anvers dans une Bible intégrale vernaculaire qui, toute mention du nom de Benoist enlevée, fut cette fois-ci acceptée par l'Église catholique et connue sous le nom de «Bible de Louvain ${ }^{78}$ ». Il est intéressant de remarquer, après cette

${ }^{74}$ La Saincte Bible, Louvain, Bartholomy de Grave, Anthoine Marie Bergagne et Jehan de Waen, 1550. Selon Chambers, cette Bible a visiblement été traduite à partir de la Bible latine publiée à Louvain en 1547, mais porte aussi l'influence des versions de Jacques Lefèvre d'Étaples de Genève.

75 « Car il ne faut point seulement croire ce qui se trouve ès saintes Ecritures, ains tout ce que l'Eglise ordonne et dispose par l'inspiration du Saint-Esprit, ou autrement n'avons rien de certain. » $c f$. La Saincte Bible, Louvain, Bartholomy de Grave et al., 1550, «Préface au lecteur». Betty Thomas Chambers, Bibliography of French Bibles..., op. cit., p. 169. Voir encore Pierre-Maurice Bogaert et Jean-François Gilmont, «La première Bible française de Louvain (1550)», Revue théologique de Louvain, vol. 11, n 3, 1980, p. 275-309.

${ }^{76}$ Le Nouveau Testament... par M. René Benoist, Angevin, Docteur regent en la faculté de Theologie à Paris, Paris, Sébastien Nivelle, 1566. Selon Chambers, les errata montrent l'influence genevoise sur cette édition. La Sainte Bible... par M. René Benoist, Angevin, Docteur Regent en la faculté de Théologie à Paris, Paris, Sébastien Nivelle, 1568. La modification du titre de cette édition rend bien clair que les annotations et expositions étaient pour «ceux qui ouvertement confirment la Foy et Religion Catholique ». Richard Simon signale par ailleurs que Benoist avait beaucoup emprunté à la Bible genevoise. "On n'a point d'autre traduction française de la Bible sur le texte hébreu que celle des Docteurs de Genève [...]. René Benoist, Docteur en théologie de Paris a aussi publié une nouvelle traduction de la Bible sur les originaux, bien qu'il ne sût ni grec ni hébreu », $c f$. Richard Simon, Histoire critique du Vieux Testament, Rotterdam, Reenier Leers, 1685, p. 53.

${ }^{77}$ Victor Baroni, « Les versions pour le peuple », art. cit.

${ }^{78}$ La Saincte Bible..., Anvers, Christophe Plantin, 1578. Selon Chambers, cette Bible, qui serait le modèle de la «Bible de Louvain » et qui était en réalité une révision de la Bible de René Benoist (notamment pour l'Ancien Testament et l'Apocryphe), évitait par précaution toute mention de ce théologien. En outre, Plantin avait obtenu l'approbation de plusieurs docteurs de la Faculté de Théologie à l'Université de Louvain. On y voit les efforts de Plantin pour garantir l' «orthodoxie » absolue de l'édition. Cf. Betty Thomas Chambers, Bibliography of French Bibles..., op. cit., p. $421-423, \mathrm{n}^{\circ} 439$. 
parenthèse sur les Bibles catholiques, que les textes mêmes des Bibles à caractère catholique ou réformé empruntaient beaucoup les uns aux autres et que leurs différences ne résidaient que dans les détails ou les nuances du sens des mots. Les disputes entre les docteurs et la concurrence entre les différentes éditions de la Bible étaient donc loin d'être simplement philologiques : elles étaient aussi et surtout symboliques et politiques.

Avec les sollicitations d'un lectorat sensible aux idées réformées, la période 1562-1567 fut donc propice à la publication de Nouveaux Testaments vernaculaires genevois. Benoît Rigaud, par exemple, qui n'avait jamais publié de Bible auparavant, fit paraître en 1566 son premier et unique Nouveau Testament genevois. Il s'était d'ailleurs converti au calvinisme durant cette période, avant d'opérer une reconversion rapide au catholicisme dès $1567^{79}$. La posture de Roville vis-à-vis des autorités politico-religieuses était plus prudente ${ }^{80}$. En outre, en 1567, lors de la première participation des imprimeurs-libraires aux élections consulaires, Roville fut élu premier syndic ${ }^{81}$ des imprimeurs-libraires et figura ensuite au Consulat ${ }^{82}$. En raison de sa grande influence et de son statut élevé, il ne jouissait probablement pas de la même liberté que les autres libraires lorsqu'il s'agissait de définir sa politique éditoriale. Il devait être prudent afin de conserver un statut privilégié et des responsabilités au sein de la communauté civile. La visibilité que lui conférait ce statut le rendait également plus exposé aux attaques et aux critiques.

\footnotetext{
${ }^{79}$ Natalie Zemon Davis, «On the Protestantism of Benoît Rigaud », Bibliothèque d'Humanisme et Renaissance, $\mathrm{n}^{\circ} 17$ (2), 1955, p. 246-251.

${ }^{80}$ Henri et Julien Baudrier, Bibliographie lyonnaise..., op. cit., vol. 7, p. 74-75.

${ }^{81}$ Les bourgeois lyonnais avaient l'habitude d'appeler leurs représentants les «syndics ».

${ }^{82}$ Henri et Julien Baudrier, Bibliographie lyonnaise..., op. cit., vol. 7, p. 74-75.
} 


\section{La publication des textes bibliques illustrés}

Les textes bibliques illustrés constituent un autre aspect majeur de la production de Roville à partir des années 1562-1563. Le libraire publia en 1570 un Nouveau Testament français en huitains et illustré d'images, ainsi que deux autres versions illustrées de ce texte en 1570 et 1588, en vers italiens. Le format est in-octavo (in-8), assez facile à porter. Ce qui conduisit Roville à publier ces éditions transparaît dans la dédicace aux lecteurs figurant en tête des Figures du Nouveau Testament de $1570^{83}$. Après avoir fait référence à la Réforme, qu'il qualifie de «peste », «grande» et «contagieuse », qui l'aurait empêché de travailler sur les figures du Nouveau Testament, Roville explique en détail la genèse de cette publication ainsi que la manière dont il pense répondre aux attentes de sa clientèle. En annonçant sa volonté de « servir au commun », Roville fait probablement allusion à ses responsabilités civiques, puisqu'il était alors capitaine pennon du quartier ${ }^{84}$. La mention de ses nombreux «amys » lettrés ou peintres suggère aussi qu'il possédait un réseau professionnel particulièrement étendu.

La Bible illustrée n'était pas une nouveauté. On peut en effet comparer cette préface avec celle rédigée par Jean I de Tournes, figurant au début des Quadrins historiques de la Bible, une édition illustrée parue en $1555^{85}$. Selon de Tournes, le but de sa «representacion de la sainte Bible » est de bien faire connaître le contenu de l'Ancien Testament par

\footnotetext{
${ }^{83} \ll[\ldots]$ ie me suis resolu de passer outre à faire pourtraire \& tailler [...] les figures du Nouueau testament, de la grandeur \& proportion de celles de la Bible, lesquelles n'ont iamais esté veües, ne imprimeés qu'à present. Ie les ay aussi d'abondant faict illustrer de huictains, mis au pied pour l'explication de chaque figure, sachant bien que la paincture \& la poësie ont grande affinité ensemble. »Cf. Figures du Nouveau Testament Illustrées de huictains Françoys, pour l'interprétation et intelligence d'icelles, Lyon, Guillaume Roville, 1570, «Aux lecteurs ».

${ }^{84}$ Henri et Julien Baudrier, Bibliographie lyonnaise..., op. cit., vol. 7, p. 74-75.

${ }^{85}$ Quadrins historiques de la Bible, Lyon, Jean de Tournes, 1555.
} 
l'image $^{86}$. On constate ainsi une légère différence entre le catholique Roville, qui met l'accent sur l'effet que produit la combinaison texteimage, et le réformé de Tournes qui considère son édition comme un substitut de l'Écriture au cas où sa lecture ne serait pas possible ${ }^{87}$.

La posture de Roville concernant la publication des textes bibliques reflète assez bien celle d'une partie des libraires lyonnais. D'une part, le statut de la Bible intégrale latine fut maintenu voire renforcé suite au Concile de Trente, au détriment des Nouveaux Testaments vernaculaires ; d'autre part, il y eut une croissance significative de la publication de textes bibliques, versifiés ou illustrés, destinés à toucher un plus grand public.

On peut observer, à travers Roville et l'évolution de sa politique éditoriale, que le Concile de Trente et la mouvance de la Contre-Réforme exercèrent progressivement une pression sur les libraires lyonnais. La publication de la Bible était donc chose délicate. Eux-mêmes lecteurs et vivant dans un espace urbain, les libraires lyonnais étaient particulièrement susceptibles d'être influencés par les idées réformées, en raison de leur participation au commerce des livres; plusieurs furent d'ailleurs séduits par ces nouvelles idées. Cela n'équivalait pourtant pas toujours à une rupture totale avec l'Église catholique. Le souci du succès commercial était aussi une grande priorité. Tous ces facteurs firent qu'une grande hésitation entre les confessions fut caractéristique de beaucoup de libraires lyonnais. La Contre-Réforme encourageait l'orthodoxie de la Vulgate et la reconquête catholique, ce qui induisit d'importants

\footnotetext{
${ }^{86}$ « $[\ldots]$ cetui labeur, qui est la representacion de la sainte Bible [...]. [...] tout le vieil testament n'estoit que l'image, et figure de celui [...] : à celle fin aussi que, ayant souvent devant tes yeus l'histoire de la vie des saints Patriarches, tu puisses si bien conformer la tienne à leur exemple [...]. », cf. ibid., «L'imprimeur au lecteur ».

${ }^{87}$ «[...] i'ay tasché de te plaire en cetui labeur, qui est la representacion de la sainte Bible, à celle fin que, si tu n'as pas le loisir de lire et iouir de la lettre comme tu desirerois, tu puisses pour le moins tapisser les chambres de ta mémoire des figures d'icelle », $c f$. ibid.
} 
changements au sein de la librairie et au cœur de l'Église même. En imposant une ligne éditoriale aux libraires, l'Église catholique connut graduellement une transformation. En quelques décennies, sous le choc de la Réforme, l'Église catholique passa du rejet de l'imprimerie à l'encouragement des Bibles latines, d'une diffusion essentiellement orale d'histoires bibliques à une diffusion largement écrite et souvent facilitée par les illustrations et les vers. L'évolution de la politique éditoriale du libraire lyonnais Guillaume Roville constitue un exemple privilégié pour observer de près la naissance progressive d'une littérature proprement «catholique », parallèle aux processus de la «construction confessionnelle» et de la «confessionalisation» au cours du $\mathrm{XVI}^{\mathrm{e}}$ siècle $^{88}$. Comme ce double processus, cette littérature « catholique » contribue à renforcer l'appartenance confessionnelle auprès de ses promoteurs et à approfondir les divergences entre le catholicisme et les nouvelles croyances.

88 Pour une présentation de la notion de «construction confessionnelle » (Konfessionsbildung) inventée par les historiens allemands, et pour une explication du double processus, voir Thierry Wanegffelen, "Construction confessionnelle" et "confessionalisation" dans l'Europe moderne », Historiens et géographes, n 341, octobre 1993, p. 121-132. 
